\title{
Heavy-Light Scalar and Axial-Vector Mesons Decay Constants and Masses in QCD Sum Rule Approach
}

\section{J.Y. Süngü ${ }^{*}{ }^{a}$ H. Sundu ${ }^{b}$, K. Azizi ${ }^{c}$, N. Yinelek ${ }^{d}$ and S. Şahin ${ }^{e}$}

a $b$ d e Department of Physics, Kocaeli University, 41380, Izmit, Turkey

${ }^{c}$ Physics Division, Faculty of Arts and Sciences, Dŏ̆us University, Acıbadem-Kadıköy, 34722

Istanbul, Turkey

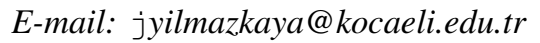

E-mail: ఐayriye.sundu@kocaeli.edu.tr

E-mail: 四azizi@dogus.edu.tr

E-mail: 四eseyinelek@gmail.com

E-mail: ssinemsahin-87@hotmail.com

\begin{abstract}
Many new results on heavy-light decay constants and masses have been obtaining recently thanks to advanced experimental techniques and searches. These experiments have learned a lot from states made of both light and heavy quarks. In the present work, the decay constants and masses of heavy-light mesons have been evaluated using QCD sum rules method. We compare obtained results of scalar and axial-vector mesons decay constants and masses with the predictions of the other approaches and experiments.
\end{abstract}

PACS Numbers 11.55.Hx, 14.40.-n, 14.40.Ev.

The Many Faces of $Q C D$

November 2-5, 2010

Gent, Belgium

\footnotetext{
* Speaker.
} 


\section{Introduction}

Reliable estimates of heavy-light meson decay constants are of particular theoretical and experimental importance, since they appear in many processes from which fundamental quantities can be extracted. They provide a precise determination of the overlap of the wave functions of the heavy and light quarks within the meson. It is represented by a single decay constant for each meson species. This direct approach, however, meets several difficulties. First of all, decay constant $f_{D}$ and $f_{B}$ can in principle be extracted from decays of charged $D$ and $B$ mesons, respectively. The corresponding decay width, namely decay probability of decay is

$$
\begin{gathered}
\Gamma\left(D_{s}^{+} \rightarrow l^{+} v_{l}\right)=\frac{G_{F}^{2}\left|V_{c s}\right|^{2}}{8 \pi} f_{D_{s}}^{2} m_{l}^{2} m_{D_{s}}\left(1-\frac{m_{l}^{2}}{m_{D_{s}}^{2}}\right)^{2}, \\
\Gamma(B \longrightarrow l v)=\frac{G_{F}^{2}\left|V_{u b}\right|^{2}}{8 \pi} f_{B}^{2} m_{l}^{2} m_{B}\left(1-\frac{m_{l}^{2}}{m_{B}^{2}}\right)^{2}
\end{gathered}
$$

where $G_{F}$ is Fermi constant, $m_{l}$ the lepton mass, $m_{D_{s}}$ the mass of $D$ meson and $m_{B}$ the mass of $B$ meson. This width is seen to be quite small due to the smallness of the CKM factor $\left|V_{u b}\right|,\left|V_{c s}\right|$ and helicity suppression factor of $m_{l}^{2}$. The total decay width of $D$ and $B$ meson is related to its mean life $\tau$ which plays a crucial role in the extraction from experimental data of CabibboKobayashi-Maskawa (CKM) matrix elements, quark mixing and $C P$ violation parameters as well as outstanding opportunities for investigating new physics. To determine these hadronic parameters precisely, many experimental attempts has been made such as BaBar, CLEO and BELLE Collaborations [-3].

In this paper, we calculate decay constants and masses of heavy-light scalar mesons with quantum numbers $J^{P}=0^{+}$and axial-vector mesons with the quantum numbers $J^{P}=1^{+}$, within the framework of the two-point QCD sum rules. In the literature there has been various nonperturbative approaches such as Instanton models, Lattice simulation of QCD, Nambu-jona-lasinio model (NJL), Heavy quark effective theory (HQET), Relativistic quark models, Chiral perturbation theory, Green function approach, Vector meson dominance model, etc. Among them QCD sum rules (QCDSR) has become very powerful and informative tool in hadron phenomenology in the nonperturbative region.

The rest of the paper is organized as follows. In Section II, we obtain the QCD sum rules for scalar and axial-vector meson decay constants and masses. In Section III, we present our numerical results and comparison with lattice theory, experiment and the other methods.

\section{Sum Rules for the Decay Constants and Masses of the Scalar and Axial-Vector Mesons}

The method of QCD sum rules developed by Shifman, Vainshtein and Zakharov (SVZ) in 1979 provides an efficient method to investigate hadronic observables, such as decay constants, masses, coupling constants and form factors, in terms of nonperturbative contributions proportional to the 
quark and gluon condensates 4 . In the QCDSR method, hadrons are represented by their interpolating quark currents. The correlation function of these currents is treated within the framework of the Operator Product Expansion (OPE), where the short and long distance quark-gluon interactions are separated. The QCDSR is constructed from the two-point correlation function. In the following, we write down the two-point correlation functions $\Pi_{S}(q)$ and $\Pi_{A V}^{\mu v}(q)$

$$
\begin{gathered}
\Pi_{S}(q)=i \int d^{4} x e^{i q \cdot x}\left\langle 0\left|T\left[j_{S}(x) \bar{j}_{S}(0)\right]\right| 0\right\rangle \\
\Pi_{A V}^{\mu v}(q)=i \int d^{4} x e^{i q \cdot x}\left\langle 0\left|T\left[j_{A V}^{\mu}(x) \bar{j}_{A V}^{v}(0)\right]\right| 0\right\rangle
\end{gathered}
$$

where $\mathrm{T}$ is the time ordered product, $q$ is the four-momentum and $j_{S}(x)=\bar{q}_{1}(x) q_{2}(x), j_{A V}^{\mu}(x)=$ $\bar{q}_{1}(x) \gamma_{\mu} \gamma_{5} q_{2}(x)$ is a possible quark current describing the scalar mesons and axial-vector mesons state, respectively. Correlation function can be evaluated in two ways:

- In the QCD or theoretical side written in terms of quark degrees of freedom, the correlators are evaluated using the Wilson Operator Product Expansion. The OPE in QCD is a double expansion: a perturbative expansion in powers of the strong coupling $\alpha_{s}$ and an expansion in powers of the Borel parameter in terms of condensates of increasing dimension.

- The phenomenological representation or physical side written in terms of hadronic degrees of freedom of the sum rule is responsible for the introduction of the decay constants and masses.

First, let us start to compute the physical side. According to the basic assumption of quarkhadron duality in the QCD sum rules approach 团, we can insert a complete series of intermediate states with the same quantum numbers as the current operators $j_{S}(x), j_{A V}^{\mu}(x)$ into the correlation functions $\Pi_{S}(q)$ and $\Pi_{A V}^{\mu v}(q)$ and obtain the hadronic representation.

$$
\begin{gathered}
\Pi_{P h y(S)}(q)=\frac{\langle 0|j| S\rangle\langle S|j| 0\rangle}{m_{S}^{2}-q^{2}}+\ldots \\
\Pi_{P h y(A V)}^{\mu v}(q)=\frac{\left\langle 0\left|j_{A V}^{\mu}(x)\right| A V(q, \varepsilon)\right\rangle\left\langle A V(q, \varepsilon)\left|\bar{j}_{A V}^{v}(0)\right| 0\right\rangle}{m_{A V}^{2}-q^{2}}+\ldots
\end{gathered}
$$

here the $\varepsilon^{\mu}$ is the polarization vector. Decay constants are defined by the matrix elements of currents between the meson states and the vacuum. Therefore, in order to calculate them, one has to find a way to evaluate hadronic matrix elements. We have employed the standard definitions of the decay constants $f_{S}$ and $f_{A V}$,

$$
\begin{gathered}
\left\langle 0\left|J_{S}(0)\right| S \mid\right\rangle=f_{S} m_{S} \\
\left\langle 0\left|J_{A V}^{\mu}(0)\right| A V(q, \varepsilon)\right\rangle=f_{A V} m_{A V} \varepsilon^{\mu}
\end{gathered}
$$


The correlation function for heavy-light scalar mesons can be expanded in terms of OPE at large negative value of $q^{2}$ :

$$
\Pi_{P h y(S)}(q)=\frac{f_{S}^{2} m_{S}^{2}}{m_{S}^{2}-q^{2}}+\ldots
$$

The correlation function of axial-vector mesons $\Pi_{P h y(A V)}^{\mu v}(q)$ can be decomposed as follows:

$$
\Pi_{P h y(A V)}^{\mu v}=\frac{f_{A V}^{2} m_{A V}^{2}}{m_{A V}^{2}-q^{2}}\left(-g_{\mu v}+\frac{p_{\mu} p_{v}}{m_{A V}^{2}}\right)+\ldots
$$

where the dots denote higher resonance contributions that will be parameterized, as usual, through the introduction of the continuum threshold parameter $s_{0}$.

Secondly, in QCD side, the correlation function is calculated in deep Euclidean region, $q^{2} \ll$ $-\Lambda_{Q C D}^{2}$ via OPE where the short or perturbative and long distance or non-perturbative effects are separated, i.e.,

$$
\Pi^{Q C D}(q)=\Pi^{\text {pert }}(q)+\Pi^{\text {nonpert }}(q)
$$

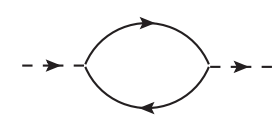

(a)

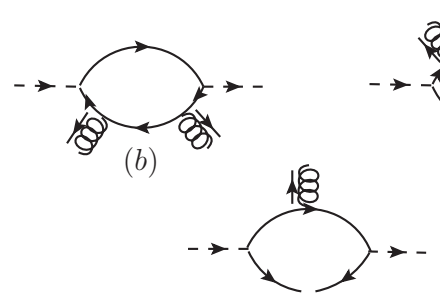

(e)

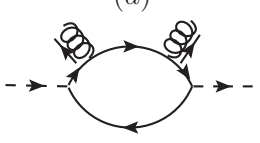

(c)
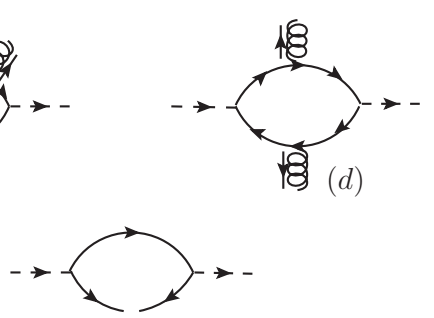

$(f)$

Figure 1: Possible diagrams for the heavy-light scalar and axial-vector mesons up to dimension five, corresponding to bare loop (a), gluon condensates (b, c, d), quark-gluon mix condensates (e) and quark condensate contributions (f) to the correlation function.

The short distance contribution (i.e. bare loop diagram in fig. (1) part (a)) is computed using the perturbation theory, whereas the long distance contributions (diagrams shown in fig. (1) part (b),(c),(d),(e)and (f)) are represented in terms of the vacuum expectation values of the operators having different mass dimensions. We can write the perturbative part in terms of a dispersion integral,

$$
\Pi^{O P E}\left(q^{2}\right)=\int_{0}^{\infty} \frac{d s}{\left(s-q^{2}\right)} \rho(s)+\Pi^{\text {nonpert }}
$$


where the spectral density is given by the imaginary part of the correlation function: $\rho(s)=$ $\frac{1}{\pi} \operatorname{Im}\left[\Pi^{O P E}(s)\right][5-6$. After some straightforward calculations, we obtain the spectral density for the heavy-light scalar mesons

$$
\rho(s)=\frac{N_{c}}{4 \pi^{2} s}\left(m_{1}^{2}+m_{2}^{2}-s+2 m_{1} m_{2}\right) \sqrt{\left(s+m_{1}^{2}-m_{2}^{2}\right)^{2}-4 s m_{1}^{2}}
$$

and for the heavy-light axial-vector mesons

$$
\begin{gathered}
\rho^{\mu v}(s)=\frac{N_{c}}{\pi^{2}} \sqrt{\frac{\left(m_{1}^{2}-m_{2}^{1}+s\right)^{2}}{4 s}}-m_{1}\left[P_{\mu} P_{v} \rho_{1}(s)+g_{\mu \nu} \rho_{2}(s)\right] \\
\rho_{1}(s)=\left(\frac{2\left(m_{1}^{2}-m_{2}^{2}+s\right)^{2}}{3 s^{2}}-\frac{m_{1}^{2}-m_{2}^{2}+s}{s}-\frac{2 m_{1}^{2}}{3 s}\right) \\
\rho_{2}(s)=\left(\frac{m_{1}^{2}-m_{2}^{2}+s}{2}-\frac{m_{1}^{2}}{3}-m_{1} m_{2}-\frac{\left(m_{1}^{2}-m_{2}^{2}+s\right)^{2}}{6 s}\right)
\end{gathered}
$$

where $m_{1}$ and $m_{2}$ are the masses of the upper and lower quarks of the loop, respectively and $N_{c}=3$ is the number of color.

Applying Borel transformation, all subtraction terms vanish and the resulting sum rule transforms into a simple relation for decay constant and mass:

$$
\begin{aligned}
& f^{2} e^{-m^{2} / M^{2}}=\int_{\left(m_{1}+m_{2}\right)^{2}}^{s_{0}} d s \rho(s) e^{-s / M^{2}}+\widehat{B} \Pi^{\text {nonpert }} \\
& m^{2}=\frac{\frac{d}{d\left(-\frac{1}{M^{2}}\right)}\left[\int_{\left(m_{1}+m_{2}\right)^{2}}^{s_{0}} d s \rho(s) e^{-s / M^{2}}+\widehat{B} \Pi^{\text {nonpert }}\right]}{\int_{\left(m_{1}+m_{2}\right)^{2}}^{s_{0}} d s \rho(s) e^{-s / M^{2}}+\widehat{B} \Pi^{\text {nonpert }}}
\end{aligned}
$$

where $\widehat{B}$ represent Borel transformation. The expression for $\widehat{B} \Pi^{\text {nonpert }}$ is quite lengthy and here we do not represent its explicit form for both scalar and axial-vector mesons.

Since $m_{\text {quark }} \leq \Lambda_{Q C D}$, we treat $\mathrm{u}, \mathrm{d}$ and s quark as light quarks. Both charm and bottom quarks can be regarded as heavy quarks. While for the heavy quarks only the interactions with the vacuum gluons are important, in the case of light quarks, quark condensates are dominant $\square$. Up to dimension five, all the diagrams contributing to the correlation function are shown in Fig. 1: 
Table 1: Input parameters at $\mu=1 \mathrm{GeV}$

\begin{tabular}{lll}
\hline Parameters & Numerical Values & References \\
\hline$m_{u}$ & $0.005 \mathrm{GeV}$ & $\square$ \\
$m_{d}$ & $0.007 \mathrm{GeV}$ & $\square$ \\
$m_{s}$ & $0.14 \mathrm{GeV}$ & $\square$ \\
$m_{c}$ & $1.3 \mathrm{GeV}$ & $\square$ \\
$m_{b}$ & $4.7 \mathrm{GeV}$ & $\square$ \\
$\alpha\left(M_{Z}\right)$ & $0.119 \mathrm{MeV}$ & $\square$ \\
$\left\langle\bar{\psi} g G_{\lambda \tau}^{c} \sigma_{\lambda \tau} \psi\right\rangle$ & $m_{0}^{2}\langle\bar{\psi} \psi\rangle$ & $\square$ \\
$m_{0}^{2}$ & $0.8 \mathrm{GeV}$ & $\square$ \\
$\left\langle\frac{\alpha_{s}}{\pi} G^{2}\right\rangle$ & $0.012 \pm 0.004 \mathrm{GeV}^{4}$ & 9,4 \\
$\langle\bar{u} u\rangle=\langle\bar{d} d\rangle$ & $-(0.24)^{3} \mathrm{GeV}^{3}$ & 9,4 \\
$\langle\bar{s} s\rangle$ & $0.8\langle\bar{u} u\rangle$ & 9,4 \\
\hline
\end{tabular}

\section{Numerical Analysis}

In this section, we present the numerical analysis of the sum rules. We used numerical values in our calculations as shown in Table 1 and choose the continuum threshold as $\left(m_{\text {Hadron }}+0.3\right)^{2} \leq$ $s_{0} \leq\left(m_{\text {Hadron }}+0.7\right)^{2}$. The threshold parameter $s_{0}$ which, in general, has to be fitted, is expected to be close to the mass squared of the first excited state of scalar and axial-vector mesons. The sum rules contain also two auxiliary parameters; the continuum threshold $s_{0}$ and Borel mass parameter $M^{2}$, therefore, the physical quantities should be independent of these parameters. Hence, we search for the regions where the physical quantities are approximately independent on these parameters. The sum rules (Eq.2.15 -Eq.2.16) are not applicable at too small $M^{2}$ because the missing terms with higher-dimensional condensates and also the short-distance nonperturbative effects, all proportional to large powers of $1 / M^{2}$, may become too important to be neglected. At too large $M^{2}$ the quark-hadron duality approximation cannot be trusted. Therefore, one also has to choose an upper limit on $M^{2}$ 5-6 for the heavy-light scalar mesons most suitable range of Borel parameter is obtained as $4 \mathrm{GeV}^{2}<M^{2}<10 \mathrm{GeV}^{2}$, for the heavy-light axial-vector mesons $5 \mathrm{GeV}^{2}<\mathrm{M}^{2}<25 \mathrm{GeV}^{2}$, where the sensitivity to the duality approximation is low. From figures 2, 3, 4 and 5, we extract the values for the masses and decay constants as presented in Tables 2, 3, 4 and 5. The errors in these Tables come from variations of the continuum threshold, Borel mass parameter and other input parameters. Tables 2, 3, 4 and 5 also includes a comparison of the decay constants and masses of the scalar and axial-vector mesons. Our results on masses are in good agreement with experimental data as well as predictions of other approaches. However our predictions on decay constants differ considerably from the other approaches predictions especially for axial-vector mesons. Our results for decay constants can be checked in future experiments.

\section{Acknowledgement}

The authors thank E.V. VELIEV for his useful comments. This work is supported by Scientific and technological research council of Turkey (TUBITAK) under project No: 110T284. 
Table 2: Decay Constants of Heavy-Light Scalar Mesons in $\mathrm{MeV}$

\begin{tabular}{||c||c||c||c||}
\hline & $B_{s j}^{(5850)}$ & $D_{0}(2400)$ & $D_{s 0}(2317)$ \\
\hline Our Results & $198 \pm 45$ & $239 \pm 73$ & $283 \pm 90$ \\
\hline Lattice Theory & $229 \pm 20[10$ & - & $200 \pm 50[1]$ \\
\hline Experiment & - & - & - \\
\hline QCDSR & $240 \pm 30[12$ & - & $201[13]$ \\
\hline
\end{tabular}

Table 3: The Decay Constants for Axial-Vector Mesons in $\mathrm{MeV}$

\begin{tabular}{||c||c||c||c||c||}
\hline & $B_{s 1}(5830)$ & $B_{1}(5721)$ & $D_{1}(2420)$ & $D_{s 1}(2460)$ \\
\hline Our Results & $572 \pm 213$ & $555 \pm 201$ & $385 \pm 175$ & $481 \pm 164$ \\
\hline $\begin{array}{c}\text { Lattice Theory } \\
\left(N_{f}=0 \text { Quenched }\right)\end{array}$ & $200 \pm 2014$ & $173 \pm 23$ 14 & - & - \\
\hline Experiment & - & - & - & - \\
\hline QCDSR & $240 \pm 20$ 12 & - & - & $225 \pm 25$ 155 \\
\hline
\end{tabular}

Table 4: The Masses of the Ground State Scalar Mesons in MeV

\begin{tabular}{|c|c|c|c|}
\hline & $B_{s j}(5850)$ & $D_{0}(2400)$ & $D_{s 0}(2317)$ \\
\hline Our Results & $5716 \pm 150$ & $2278 \pm 130$ & $2353 \pm 93$ \\
\hline $\begin{array}{c}\text { Lattice Theory } \\
\left(N_{f}=0 \text { Quenched }\right)\end{array}$ & - & - & $2248 \pm 78$ [16 \\
\hline Experiment & $5853 \pm 150$ [1] & $2317.8 \pm 6 \square 0$ & $2317.8 \pm 0.6[\square]$ \\
\hline QCDSR & $5850 \pm 130$ 묘 & - & $2510 \pm 27[19]$ \\
\hline
\end{tabular}

Table 5: The Masses of the Ground States Axial-Vector Mesons in MeV

\begin{tabular}{||c||c||c||c||c||}
\hline & $B_{s 1}(5830)$ & $B_{1}(5721)$ & $D_{1}(2420)$ & $D_{s 1}(2460)$ \\
\hline Our Results & $5720 \pm 130$ & $5760 \pm 50$ & $2610 \pm 190$ & $2410 \pm 60$ \\
\hline $\begin{array}{c}\text { Lattice Theory } \\
\left(N_{f}=0 \text { Quenched }\right)\end{array}$ & - & - & - & $2476 \pm 92$ [16 \\
\hline Experiment & $5829.4 \pm 0.7[1]$ & $5723.4 \pm 2[1]$ & $2423.4 \pm 3.1[1]$ & $2459.6 \pm 6[17$ \\
\hline QCDSR & - & - & - & $2510[19$ \\
\hline
\end{tabular}

\section{References}

[1] BaBar Collaboration, B. Aubert et al., Measurement of the Pseudoscalar Decay Constant $f_{D s}$ Using Charm-Tagged Events in e+e-Collisions at $\sqrt{s}=10.58 \mathrm{GeV}$, Phys. Rev. Lett. 98 (141801) 2007 [hep-ex/0607094].

[2] CLEO collaboration, D. Besson et al., Observation of a Narrow Resonance of Mass $2.46 \mathrm{GeV} / \mathrm{c}^{2}$ Decaying to $D_{s}^{*+} \pi^{0}$ and Confirmation of the $D_{s J}^{*}(2317)$ State, Phys. Rev. D 68 (032002) 2003; Erratum-ibid.D75 (119908) 2007 [hep-ex/ 030510 0v2]. 

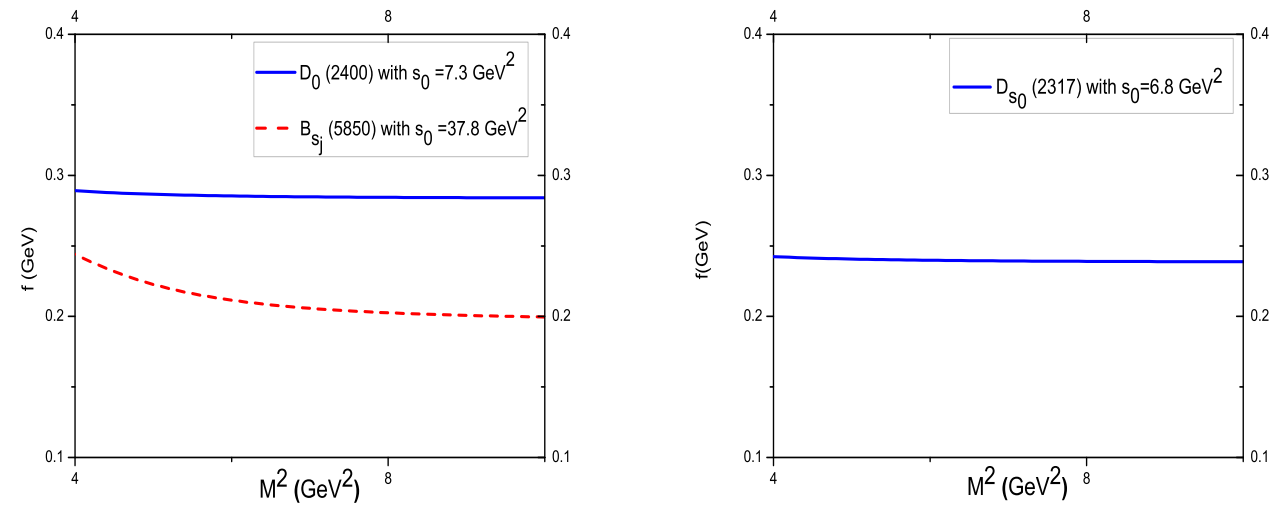

Figure 2: The dependence of the leptonic decay constant of the $D_{0}(2400), B_{s j}(5850)$ and $D_{s 0}(2317)$ as heavy-light scalar mesons on Borel mass parameter.
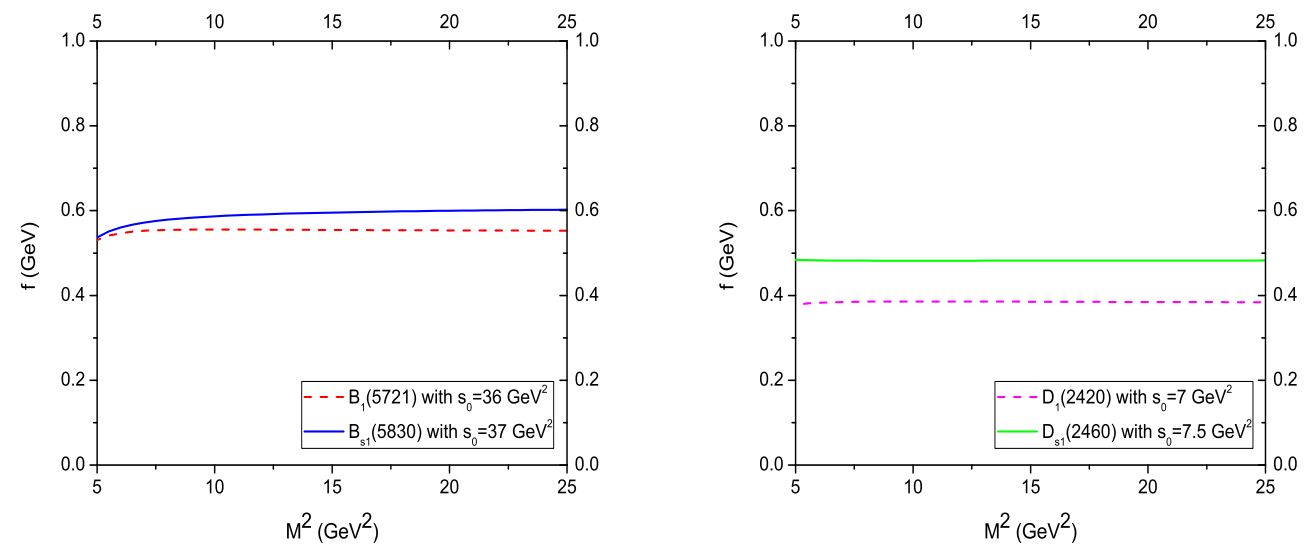

Figure 3: The dependence of the leptonic decay constant of the $B_{1}(5721), B_{s 1}(5830), D_{1}(2420)$ and $D_{s 1}(2460)$ as heavy-light axial-vector mesons on Borel mass parameter.

[3] BELLE Collaboration, P. Krokovny et al., Observation of the $D_{s J}(2317)$ and $D_{s J}(2457)$ in B Decays, Phys. Rev. Lett. 91 (262002) 2003 [hep-ex/0308019v3].

[4] M. A. Shifman, A.I. Vainshtein and V.I. Zakharov, QCD and Resonance Physics. Theoretical Foundations, Nucl. Phys. B 147 (385) 1979; M.A.Shifman, A.I. Vainshtein and V.I.Zakharov, QCD and Resonance Physics. Applications, Nucl. Phys. B 147 (448) 1979.

[5] P. Colangelo, A. Khodjamirian, QCD Sum Rules, A Modern Perspective At the Frontier of Particle Physics Handbook of QCD, edited by M. Shifman (World Scientific, Singapore) 3 (1495) 2001 [hep-ph/0010175].

[6] L. J. Reinders, S. Yazaki and H. R. Rubinstein, Hadron Properties from QCD Sum Rules, Phys. Rep.127 (1) 1985. 

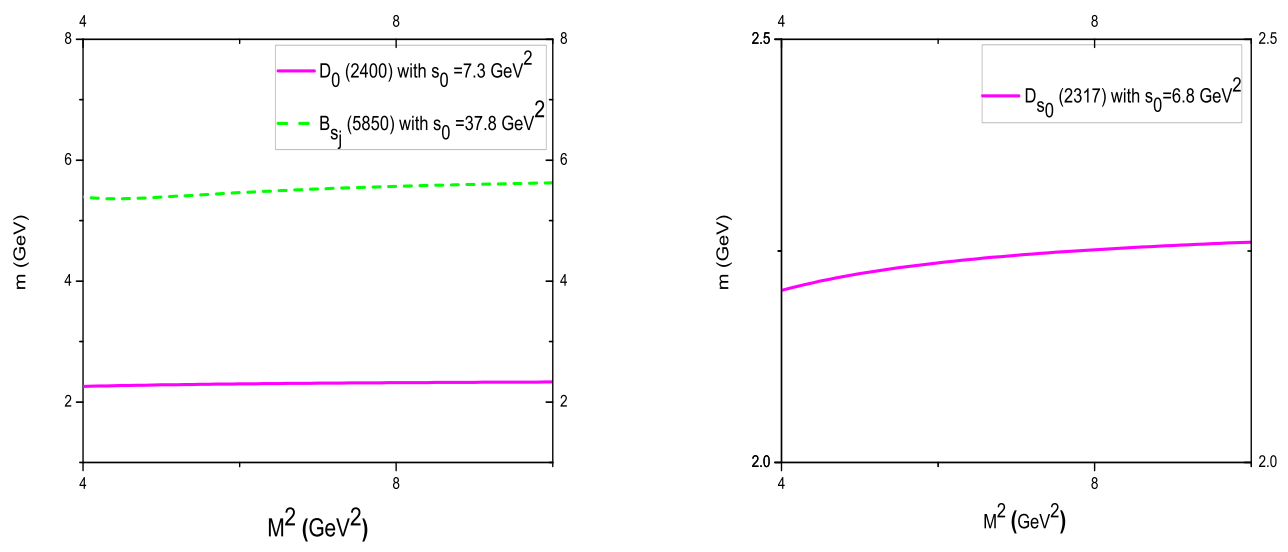

Figure 4: The dependence of the mass of the $D_{0}(2400)$ and $B_{s j}(5850)$ and $D_{s 0}(2317)$ as heavy-light scalar mesons on Borel mass parameter.
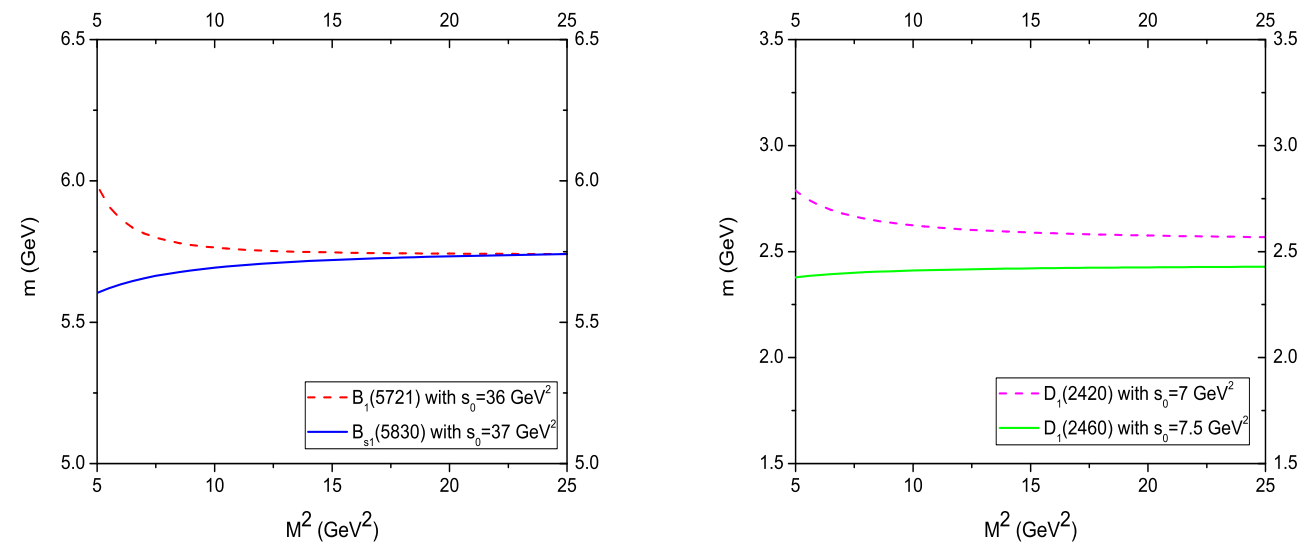

Figure 5: The dependence of the mass of the $B_{1}(5721) B_{s 1}(5830) D_{1}(2420)$ and $D_{s 1}(2460)$ as heavy-light axial-vector mesons on Borel mass parameter.

[7] C. Amsler et al., (Particle Data Group), Note On Scalar Mesons, Phys. Lett B 667 (1) 2008; Revised version in PDG 2010.

[8] S. Bethke, The 2009 World Average of $\alpha_{s}$, Eur. Phys. J. C 64(689) 2009 [hep-ph/ 0908 .1135v2].

[9] V. M. Belyaev, B. L. Ioffe, Determination Of Baryon And Baryonic Masses From Qcd Sum Rules. Strange Baryons. , Sov. Phys. JETP 57 (716) 1982; B. L. Ioffe, QCD at Low EnergiesProg. Part. Nucl. Phys. 56 (232) 2006 [hep-ph/ 0502148$].$

[10] D. Becirevic et al., Non-perturbatively Improved Heavy-Light Mesons : Masses and Decay Constants, Phys. Rev. D 60 (074501) 1999 [hep-lat/9811003].

[11] G. Herdoiza, C. McNeile, C. Michael, Decay Constants of P-wave Heavy-light Mesons from Unquenched Lattice QCD, Phys. Rev. D 74 (014510) 2006 [hep-lat/ 0604001 ]. 
[12] Z. G. Wang, Reanalysis of the $\left(0^{+}, 1^{+}\right)$States $B_{s 0}$ and $B_{s 1}$ with QCD Sum Rules, Chin. Phys. Lett. 25 (11) 39082008 [hep-ph/0712.0118].

[13] S. Narison, Open Charm and Beauty Chiral Multiplets in QCD, Phys. Lett. B 605 (319) 2005 [hep-ph/0307248].

[14] S. Hashimoto, T. Onigi, Heavy Quarks on the Lattice, Ann. Rev. Nucl. Part. Sci., 54 (451) 2004 [hep-ph/0407221].

[15] Z. G. Wang, Radiative decays of the $D_{s 0}(2317), D_{s 1}(2460)$ and the related strong coupling constants, Phys. Rev. D 25 (034013) 2007 [hep-ph / 0612225 v 4].

[16] S.J. Dong et al., The charmed-strange meson spectrum from overlap fermions on domain wall dynamical fermion configurations, in proceedings of The XXVII Inter. Symp. on Lat. Field Theo. July 26-31 Peking University, Beijing, China,

[17] K. Nakamura et al., (Particle Data Group), Review of Particle Physics, J. Phys. G 37 (075021) 2010.

[18] M.E. Bracco et. al., The $B_{s 0}$ meson and the $B_{s 0} B K$ coupling from $Q C D$ sum rules [hep-ph/1002.4990v2].

[19] Y. Dai et. al., Understanding the $D_{s J}^{+}(2317)$ and $D_{s J}^{+}(2460)$ with Sum Rules in HQET, Phys.Rev. D 68 (114011) 2003 [hep-ph/0306274v4]. 